\title{
Implementación de la Política Nacional de Medicamentos de la Reforma de la Salud: percepción del profesional químico farmacéutico
}

\author{
PAULINA NÚÑEZ ${ }^{(1)}$ y CLAUDIO MÉNDEZ ${ }^{(2)}$
}

\section{RESUMEN}

Introducción: La reforma de la salud en Chile incluyó una Política Nacional de Medicamentos que posiciona al profesional químico farmacéutico en la tarea de contribuir a lograr los objetivos terapéuticos y de uso racional de los medicamentos. La investigación tuvo el objetivo de explorar las percepciones de químico farmacéuticos respecto a la implementación de los cambios introducidos por esta Política. Material y Método: Se condujo un estudio cualitativo y exploratorio en base a entrevistas individuales semi-estructuradas en profundidad a profesionales químico farmacéuticos de la red asistencial de la Región Metropolitana durante el período marzo-agosto de 2010. Se seleccionó una muestra por conveniencia de 10 profesionales de diferentes niveles de atención de la red asistencial de la Región. El análisis de las entrevistas se realizó mediante la técnica de análisis de contenido. Resultados: Para los entrevistados, la Política Nacional de Medicamentos ha permitido asignar un rol importante al farmacéutico y a la farmacia dentro de las distintas instancias de la red asistencial. No obstante, las principales dificultades en su implementación han estado determinadas por la ausencia de condiciones organizacionales y estructurales de las instituciones relacionadas con la implementación de la reforma de la salud. Discusión: La implementación de la Politica Nacional de Medicamentos ha permitido mejorar algunos procesos de la gestión farmacéutica. Sin embargo; las brechas entre el diseño y la implementación de la reforma de la salud ha obstaculizado su implementación.

Palabras clave: Politica nacional de medicamentos, reforma de la salud, politicas en salud, Chile

\section{ABSTRACT}

IMPLEMENTATION OF THE HEALTH REFORM'S NATIONAL MEDICATION POLICY: THE PERCEPTION OF PHARMACISTS

Introduction: Health reform in Chile included a National Medication Policy that positioned pharmacists as contributors to achieving therapeutic goals, and as promoters of the rational use of medications. The objective of the research was to explore the perceptions of pharmacists with respect to the implementation of changes introduced by the National Medication Policy. Materials and Methods: A qualitative and exploratory study, based on individual, semi-structured, in-depth interviews with pharmacists in the Metropolitan health care network, was carried out during March-August of 2010. A convenience sample of 10 professionals was selected from different health care levels within the Metropolitan network. The analysis of the interviews was carried out using content analysis. Results: For the interviewees, the National Medication Policy has given pharmacists and pharmacies an important role in different sectors of the health care network. Nevertheless, the principal

(1) Escuela de Química y Farmacia. Facultad de Ciencias. Universidad Austral de Chile. Valdivia. Chile.

(2) Instituto de Salud Pública. Facultad de Medicina. Universidad Austral de Chile. Avenida Senador Carlos Acharán Arce, Campus Isla Teja. Valdivia. Chile.

claudiomendez@uach.cl 
difficulties in its implementation have been determined by the absence of different organizational and structural conditions in the institutions related to the implementation of the health reforms. Discussion: The implementation of the National Medication Policy has improved some processes of pharmaceutical management. Nevertheless, the gaps between the design and implementation of health reform have hampered its implementation.

Key words: National Medication Policy, health reform, health policy, Chile.

\section{INTRODUCCIÓN}

La reforma de la salud de la presente década en Chile introdujo una nueva organización del sistema de salud a través de la definición de la función rectora, el planteamiento de un nuevo modelo de atención basado en redes de salud y el establecimiento de un Régimen de Garantías en Salud en cuanto al acceso, oportunidad de atención, protección financiera y calidad respecto de un grupo de problemas de salud $^{1,2}$.

En la terapia con medicamentos, la reforma diseñó una Política Nacional de Medicamentos cuyo propósito se orientó al aseguramiento y acceso de toda la población a medicamentos de eficacia, de calidad garantizada, seguros y de costo asequible y cuyo uso racional lleve a conseguir los máximos beneficios en la salud de las personas ${ }^{3}$. En el mismo sentido, las directrices de la Política fueron el garantizar el acceso y la disponibilidad, la calidad, seguridad y eficacia de los medicamentos -principalmente genéricos- contenidos en el Formulario Nacional, junto con la racionalidad en su uso ${ }^{3}$.

En Chile, el profesional con mayores competencias en formación para la gestión farmacéutica en el sector de la salud es el químico farmacéutico. La Política Nacional de Medicamentos de la reforma estableció que el papel de este profesional estaría dirigido a la contribución del logro de los objetivos terapéuticos $\mathrm{y}$ al uso racional de los medicamentos ${ }^{3}$.

Actualmente no existen investigaciones publicadas que presenten evidencia respecto de la implementación de la Política Nacional de Medicamentos de la reforma ni se dispone de evidencia acerca de las percepciones de un profesional clave en la implementación de los cambios, como lo es el químico farmacéutico.

La presente investigación tuvo por objetivo explorar las percepciones de los profesionales químico farmacéuticos respecto de la implementación de los cambios introducidos por la Política Nacional de Medicamentos de la reforma de la salud en la red asistencial pública de la Región Metropolitana.

\section{MATERIAL Y MÉTODO}

Se condujo un estudio de tipo cualitativo, descriptivo y exploratorio en base a entrevistas semi-estructuradas individuales en profundidad a profesionales químico farmacéuticos de la red asistencial pública de salud de la Región Metropolitana. Ésta se localiza en la zona cen-

Tabla 1. Características de la muestra.

\begin{tabular}{|c|c|c|}
\hline Afiliación institucional & Función & Número entrevistados \\
\hline Hospitales de alta complejidad & $\begin{array}{c}\text { Gestión farmacéutica en las unidades de farmacia } \\
\text { hospitalaria. }\end{array}$ & 6 \\
\hline Institutos de especialidades & $\begin{array}{c}\text { Gestión farmacéutica en las unidades de farmacia } \\
\text { hospitalaria. }\end{array}$ & 1 \\
\hline Servicios de salud & $\begin{array}{c}\text { Coordinación y asesoría técnica de las farmacias } \\
\text { de la red asistencial. }\end{array}$ & 1 \\
\hline Secretaria Regional Ministerial & $\begin{array}{c}\text { Formalización, autorización sanitaria y fiscaliza- } \\
\text { ción de establecimientos farmacéuticos. }\end{array}$ & 10 \\
\hline Total entrevistados & & \\
\hline
\end{tabular}


Implementación de la Política Nacional de Medicamentos de la Reforma de la Salud - Paulina Nuñez et al

Tabla 2. Guía de entrevista semi-estructurada en profundidad.

\title{
Tópicos
}

\begin{abstract}
¿Qué papel jugó usted en el proceso de implementación de esta reforma de salud?
¿Cuál ha sido su participación profesional en la implementación de los cambios introducidos por la reforma de la salud?

¿Cómo evalúa usted la etapa de implementación de la reforma de la salud?

¿Enfrentó usted algún tipo de resistencia en el momento del cambio de gestión farmacéutica? ¿De parte de quién o quiénes?

¿Considera usted que la implementación de la reforma de la salud ha facilitado la gestión farmacéutica? ¿En qué aspectos?

¿Considera usted que la nueva política de medicamentos ha venido a fortalecer la gestión farmacéutica? ¿En qué forma?

¿Cuáles han sido los logros en la gestión farmacéutica desde la puesta en marcha de la Política Nacional de Medicamentos?

¿Cuáles han sido las dificultades para la gestión farmacéutica desde la puesta en marcha de la Política Nacional de Medicamentos?

¿Cómo evalúa usted el nivel de participación del profesional químico farmacéutico en la implementación de la Política Nacional de Medicamentos?

¿Cómo evalúa usted el desempeño del personal químico farmacéutico en la implementación de la Política

Nacional de Medicamentos?
\end{abstract}

tral del país y concentra alrededor del $40 \%$ de la población de Chile.

La red asistencial pública de salud de la Región está integrada por seis servicios de salud: Norte, Sur, Sur-Oriente, Oriente, Occidente y Central. Éstos son los responsables de articular y desarrollar la red asistencial compuesta por hospitales de baja, mediana y alta complejidad, centros de atención primaria de salud de administración municipal y organizaciones privadas en convenio. Por otra parte, la red se complementa con la Secretaría Regional Ministerial, la cual es la Autoridad Sanitaria Regional.

Se seleccionó una muestra por conveniencia de 10 profesionales químico farmacéuticos que se desempeñan en actividades directivas y asistenciales. Este tipo de muestra involucra la selección de personas que se encuentran disponibles o tienen la voluntad de participar de la investigación ${ }^{4}$. Los profesionales fueron contactados en sus instituciones por el primer autor, previa solicitud de autorización a las autoridades administrativas respectivas. Hubo rechazo de 4 profesionales a participar de la investigación, los cuales adujeron como causas la falta de tiempo, aprensiones a la confiden- cialidad de la información y desconocimiento del tópico de investigación. El total de la muestra correspondió a profesionales de sexo femenino con una media de edad de 43 años. Las características de la muestra se presentan en la Tabla 1.

El trabajo de campo se realizó entre los meses de marzo a agosto de 2010. Para la recolección de la información se utilizó la técnica cualitativa de entrevista semi-estructurada en profundidad $^{5}$. Se realizaron 10 entrevistas a través de las cuales se exploraron las dimensiones relacionadas a la implementación de la reforma de la salud y a la Política Nacional de Medicamentos de la reforma (Tabla 2); se buscó la saturación global de las dimensiones previamente descritas, lo cual determinó el tamaño muestral final. Las entrevistas tuvieron una duración mínima de 20 minutos y máxima de 1 hora; todas se realizaron en las dependencias de las instituciones laborales de los entrevistados. Las entrevistas fueron conducidas sólo por el primer autor, con el fin de obtener consistencia y reducir la variación de la aproximación a los temas presentados a los entrevistados ${ }^{6}$. Las entrevistas fueron grabadas, previo conocimiento por parte del entrevistado 
y suscripción del respectivo consentimiento informado. Las grabaciones se transcribieron en forma literal y, posteriormente, fueron enviadas a los entrevistados para obtener comentarios y/o correcciones.

Las entrevistas transcritas fueron leídas en forma reiterada por ambos investigadores y codificadas de acuerdo a la técnica de análisis de contenido ${ }^{7}$. En el caso de la presente investigación, se utilizó la aproximación convencional de análisis de contenido, la cual se basa en que las categorías de análisis emergen de las entrevistas y no de una concepción previa por parte de los investigadores ${ }^{8}$. Se seleccionaron citas literales de las entrevistas, de acuerdo al criterio de representación de los conceptos e ideas que emergieron de las entrevistas ${ }^{9}$.

La calidad del análisis de los datos se cauteló mediante la triangulación de investigado$\operatorname{res}^{10}$ y la reflexividad en el análisis, la cual se define como la interacción constante entre el investigador y el fenómeno estudiado cuyo fin es que ideas preconcebidas de los investigadores puedan ser mitigadas en el análisis de los resultados de la investigación ${ }^{11,12}$. En el caso de la presente investigación, el análisis se condujo reconociendo ideas preconcebidas de uno de los investigadores respecto al diseño e implementación de la reforma de la salud, para lo cual el análisis fue conducido en forma independiente por ambos investigadores para luego confrontar dichos análisis en uno de carácter global.

\section{RESULTADOS}

En la Tabla 3 se presentan las categorías y subcategorías temáticas que emergieron del análisis de las entrevistas.

Reforma de la salud y gestión farmacéutica: Para los entrevistados, la implementación de la reforma de la salud ha determinado un nuevo rol para el profesional químico farmacéutico. Este, los ha situado como líderes del grupo de trabajo dentro de la farmacia y como gestores de los procesos relacionados con la calidad de la gestión farmacéutica.

"...liderar los cambios a nivel de la farmacia, de generar conciencia en la gente que trabaja acá, que entiendan de qué se trata la Reforma, para dónde quiere ir y cuáles son los puntos en particular que nosotros, como farmacia, tenemos que trabajar... y estar con ellos en ese proceso ". (QF 9)

Las principales fortalezas identificadas por los entrevistados respecto de la implementación de la reforma se refieren al establecimiento de la salud como un derecho y el establecimiento de garantías en salud constitutivas de derecho. Para los entrevistados, este recono-

Tabla 3. Categorías y subcategorías temáticas.

\begin{tabular}{|c|c|}
\hline Categorías & Subcategorías \\
\hline \multirow{3}{*}{ Reforma de la salud y gestión farmacéutica } & Rol del profesional químico farmacéutico \\
\hline & Debilidades en la etapa implementación \\
\hline & Fortalezas en la etapa implementación \\
\hline \multirow{3}{*}{ Política Nacional de Medicamentos } & Rol del profesional químico farmacéutico \\
\hline & Aspectos del diseño de la Política \\
\hline & Debilidades en la implementación de la Política \\
\hline \multirow{2}{*}{$\begin{array}{l}\text { Cambios para mejorar la etapa de implementación de la } \\
\text { Política Nacional de Medicamentos }\end{array}$} & Recomendaciones organizacionales \\
\hline & $\begin{array}{l}\text { Desafíos disciplinares del químico } \\
\text { farmacéutico }\end{array}$ \\
\hline
\end{tabular}


cimiento determinó un cambio cultural en la conducta funcionaria en las instituciones de salud y un ordenamiento de la organización, que en el caso de la gestión farmacéutica se tradujo en mayores capacitaciones y la creación de protocolos de atención.

“... Yo siento que fue súper importante, o sea, en el fondo hay un cambio en el sentir. Le incorporaron el componente como que es derecho de las personas, entonces yo siento que eso es bueno... hicimos talleres locales con todos los especialistas, les enseñamos, decidimos los flujos locales y después ya partimos con las patologías". (QF 5)

Los entrevistados identifican como debilidades durante la implementación de la Reforma la ausencia de entrega de la información, principalmente en un contexto formal en donde se destacara los aspectos organizacionales que ésta involucraría para las instituciones del sector. Junto a la ausencia de información, los entrevistados destacan el escaso aumento del presupuesto para medicamentos y ausencia de cambios en la gestión de recursos humanos e infraestructura.

"...el tema del Auge fue muy controversial... muchos en realidad estaban en contra de que esto se haya implantado de un día para otro con poca organización, con poca comunicación... se nos exigió mucho o nos aumentó mucho la demanda o la labor sin ningún cambio en la estructura ni de recursos humanos ni de infraestructura". (QF 1)

Política Nacional de Medicamentos: Para los entrevistados, el diseño de la Política estuvo determinado por una perspectiva económica del área de los medicamentos y la gestión farmacéutica, siendo postergados aspectos organizacionales que involucraban un rol más determinante del profesional químico farmacéutico en el desarrollo de la Política y de la gestión farmacéutica. En el mismo sentido, para los entrevistados un aspecto central en el diseño de la Política fue la ausencia de una mayor integración de los profesionales químico farmacéuticos en el desarrollo de sus contenidos a través del aporte de sus competencias profesionales desarrolladas en la red asistencial pública de salud del país.

"La Política de Medicamentos en sus inicios va muy orientada a la parte económica, haciendo énfasis en la cantidad de dinero que se invierte en medicamentos. El tema del costo es importante, pero más quisiera saber en qué estoy gastando, lo estoy gastando bien y para lo que debe ser. Si la Política de Medicamentos de alguna manera establece a la farmacia y al farmacéutico como importantes, darle más énfasis a lo que es el farmacéutico en el uso seguro y adecuado de los medicamentos". (QF 4)

Como debilidades de la etapa de implementación de la Política, los entrevistados identifican la escasa evaluación sobre la eficacia del arsenal terapéutico, lo cual se relaciona directamente con la calidad de los medicamentos. Para los entrevistados, algunos de los medicamentos incluidos en las canastas farmacológicas de la mayoría de las patologías GES, no cumplen con las garantías de calidad y eficacia como lo indica la Política Nacional de Medicamentos. En tal sentido, para los entrevistados la situación previa se determina porque los procesos no están bajo la supervisión de un profesional con competencias para el control y vigilancia de los medicamentos.

"El químico farmacéutico tiene un rol muy importante en todo lo que es la dispensación de fármacos, la información a los pacientes, al equipo médico y eso es lo que a veces no alcanzamos a hacer (...) la parte clínica se nos va quedando un poco flaca y es en el fondo donde tú puedes ir a reforzar, mejorar las terapias, reducir los tiempos de hospitalización, ayudar al paciente en general. Uno espera que con una Política Nacional de Medicamentos también te aseguren de alguna manera la calidad de los productos, la calidad de los medicamentos y ahí hay problemas (...) Ni hablar de poder evaluar la eficacia, ese es otro tema, la eficacia en la calidad del producto". (QF 9)

Por otra parte, para los profesionales químicos farmacéuticos un aspecto fundamental es que la implementación de la Política cuente con el apoyo y visión de los directivos de las instituciones del sector de la salud para el de- 
sarrollo de nuevas herramientas de gestión y tecnologías que permitan mejorar la provisión de servicios de salud, la gestión farmacéutica y la satisfacción de los usuarios. Para los entrevistados, la implementación de la Política no podrá avanzar sin la voluntad de las autoridades sectoriales respectivas.

"Demás que han visto una oportunidad y la han querido desarrollar pero no han sido oídos a lo mejor...depende mucho de la visión del director. El punto es que la Política por sí misma no sirve sola (...) el director del hospital o el director del servicio tienen que tener la capacidad y la visión de ver si la mitad de los gastos del hospital se van en medicamentos y en insumos, se debe facilitar o dar las condiciones a la farmacia para que pueda revisar y controlar eso, pero si no se te dan las condiciones, el farmacéutico solo no puede hacer nada..." (QF 4)

Cambios para mejorar la implementación de la Política Nacional de Medicamentos: Los cambios sugeridos por los entrevistados en razón de mejorar la implementación de la Política Nacional de Medicamentos se orientaron a aspectos de calidad, relacionados directamente con sus responsabilidades como profesionales de la salud. Los entrevistados destacaron la importancia de mejorar la gestión en el abastecimiento del arsenal farmacológico con el fin de garantizar la disponibilidad de medicamentos esenciales a costos razonables y de altos estándares de calidad, seguridad y eficacia para la red de servicios públicos.

“...cuando hablamos de calidad es ahí cuando me gustaría que se incluyera activamente al farmacéutico en la toma de medicamentos, que sea más explicito y nosotros le vamos a asegurar al clínico que de alguna manera el medicamento cumple para lo que lo queremos, técnicamente y farmacológicamente. Que su uso seguro le va a traer resultados clínicos esperados." (QF 4)

Otro aspecto que recomiendan los entrevistados se relaciona con la mejora de los aspectos logísticos y administrativos que permitan compensar las falencias gerenciales y de com- petencias administrativas al momento de la implementación de los cambios introducidos por la reforma de la salud y la Política Nacional de Medicamentos. Asimismo, reconocen que si bien existe una mejora en el equipamiento, esto no ha sido transversal al área de recursos humanos en salud en donde se evidencia una brecha de profesionales químico farmacéuticos en relación a los requerimientos contenidos en la Política.

"...no alcanza el tiempo para hacer bien la parte administrativa, sobre todo en los centros donde tienen muy pocos profesionales. En realidad falta mejorar la calidad y recursos, por ejemplo computadores, el área logística".(QF 8)

Por otra parte, los desafíos que los entrevistados identifican para el profesional químico farmacéutico se relacionan con el posicionamiento profesional en las políticas y estrategias dirigidas al área farmacéutica. Además, para los entrevistados se hace relevante e indispensable incentivar una mayor participación e inserción de los profesionales en la atención primaria de salud. Para esto último, los profesionales perciben como fundamental la voluntad de las autoridades sanitarias para avanzar en instancias legales y profesionales que sustenten e incentiven a estos profesionales a asumir la gestión de las unidades de farmacia de los centros de salud.

"Nos falta todavía algo más de presencia farmacéutica en los consultorios. Que la Reforma diga que para una atención igualitaria, que no puede ser que cuando yo me atiendo en un hospital tengo asegurada la atención farmacéutica pero en un consultorio no". (QF7)

\section{DISCUSIÓN}

Los resultados de este estudio fortalecen la percepción respecto de la dificultad en el tránsito desde el diseño a la implementación de las reformas de la salud ${ }^{13}$. Más aún, en el caso de la implementación de la Política Nacional de Medicamentos esta dificultad ha sido reconocida por los profesionales químico farmacéuticos.

Similar a otros procesos de reforma, los as- 
pectos organizacionales y la ausencia de recursos humanos para llevar a cabo la implementación de la Política Nacional de Medicamentos fueron soslayados en privilegio del diseño de la política ${ }^{14-15}$. Esta ausencia en la reforma chilena representa una lección no aprendida respecto de la relación de las reformas de la salud y las políticas en recursos humanos en salud $^{16-19}$.

En el mismo sentido, las fortalezas y debilidades identificadas en el presente estudio respecto del diseño e implementación de la Política Nacional de Medicamentos se han presentado en otras experiencias de reformas de la salud que han sido acompañadas con políticas farmacéuticas en el continente. En Perú, una de las principales fortalezas para la implementación de la política de medicamentos fue la voluntad de los actores de los distintos niveles de toma de decisiones del sector ${ }^{20}$. En el caso de la implementación de la Política en Chile, este aspecto fue reconocido como una debilidad en la implementación.

Al igual que en Chile, la implementación de la política farmacéutica nacional de Colombia persiguió mejorar la equidad en el acceso a los medicamentos por parte de la población. Si bien las fortalezas de su diseño se relacionaron con el aseguramiento del acceso y equidad de los medicamentos, al igual que la experiencia chilena los obstáculos identificados se relacionaron con la exclusión de actores y la ausencia de capacidades organizaciones y competencias profesionales que permitieran una mejor implementación ${ }^{21}$.

Un hallazgo relevante de la investigación se relacionó con la necesidad de mayor integración de estos profesionales en las decisiones sectoriales relacionadas al diseño de políticas o estrategias dirigidas a mejorar la gestión farmacéutica Si bien existe participación del químico farmacéutico en los equipos de salud, estudios similares han identificado el alto costo de las remuneraciones y el desconocimiento de las competencias profesionales en el ámbito de la salud de este profesional como obstáculos para su integración a los equipos e instituciones, además de establecer una barrera para la implementación de una gestión farmacéutica de calidad ${ }^{22}$.

Para continuar avanzando en mejorar la calidad y equidad en el acceso a los medicamentos en el país, resulta fundamental mejorar los aspectos identificados por los entrevistados como necesarios para un buen desempeño de la Política Nacional de Medicamentos. Además, es indispensable disponer de mayor evidencia respecto de la implementación de las políticas públicas en el sector de la salud en Chile que permitan a los tomadores de decisiones dirigir nuevos cursos de acción en la implementación de las políticas.

Los resultados preliminares de este artículo fueron presentados en el II Congreso Chileno de Salud Pública 2010.

\section{REFERENCIAS}

1. REPÚBLICA DE CHILE, MINISTERIO DE SALUD DE CHILE. Ley $\mathrm{N}^{\circ}$ 19.937. Autoridad Sanitaria y Gestión. Santiago: Diario Oficial de la República de Chile; 2004 [citado 25 octubre de 2010]. www.bcn.cl/leyes/pdf/actualizado/221629. pdf.

2. REPÚBLICA DE CHILE, MINISTERIO DE SALUD DE CHILE. Ley $N^{\circ}$ 19.966. Régimen General de Garantías en Salud. Santiago: Diario Oficial de la República de Chile; 2004 [citado 25 octubre de 2010]. www.bcn.cl/leyes/pdf/actualizado/229834. pdf.

3. REPÚBLICA DE CHILE, MINISTERIO DE SALUD. Política Nacional de Medicamentos para la Reforma de la Salud. 2004.

4. ONWUEGBUZIE AJ, LEECH NL. A call for qualitative power analyses. Quality \& Quantity 2007; 41: 105-121.

5. DICICCO-BLOOM B, CRABTREE BF. The qualitative research interview. Medical Education 2006; 40: 314-321.

6. FITZPATRICK R, BOULTON M. Qualitative methods for assessing health care. Qual. Saf. Health Care 1994; 3: 107-113.

7. SANDELOWSKI M. Whatever happened to qualitative description? Research in Nursing \& Health 2000; 23: 334-340.

8. HSIEH H, SHANNON SE. Three approaches to qualitative content analysis. Qual Health Res 2005; 15(9): 1277-1288 
9. ULIN P, ROBINSON E, TOLLEY E. (2005). Qualitative Methods in Public Health: A field guide for applied research. Jossey-Bass 1st Ed. San Francisco EEUU. 318 páginas.

10. ONWEGBUZIE AJ, LEECH NL. Validity and qualitative research: an oxymoron? Quality \& Quantity. 2007; 41: 233-249.

11. CALDERÓN C. Criterios de calidad de la investigación cualitativa en salud (ICS): apuntes para un debate necesario. Rev Esp Salud Pública 2002; 76: 473-482.

12. MALTERUD K. Qualitative research: standards, challenges, and guidelines. Lancet 2001; 358: 48388.

13. ARTEAGA O. El difícil camino desde el diseño a la implementación de las reformas de salud. Rev Chil Salud Pública 2004; 8(1): 39-43.

14. NARASIMHAN V, BROWN H, PABLOS-MÉNDEZ A, ADAMS O, DUSSAULT G, ELZINGA G, et al. Responding to the global human resources crisis. Lancet 2004; 363: 1469-72.

15. BUCHAN J. What difference does ("good") HRM make? Human Resources for Health [Internet]. 2004 [citado 25 octubre 2010]; 2:6. Disponible en: http:// www.human-resources-health.com/content/2/1/6

16. MÉNDEZ CA. Los recursos humanos de salud en Chile: el desafío pendiente de la reforma. Rev Panam Salud Pública 2009; 26(3): 276-80.
17. MÉNDEZ CA. Reflexión sobre la planificación de los recursos humanos y la autonomía de gestión en los hospitales de Chile. Rev Esp Salud Pública 2009; 83: 371-378.

18. RIGOLI F, DUSSAULT G. The interface between health sector reform and human resources in health. Human Resources for Health [Internet]. 2003 [citado 10 noviembre 2010]; 1:9. Disponible en: http:// www.human-resources-health.com/content/1/1/9

19. BEAGLEHOLE R, DAL POZ MR. Public health workforce: challenges and policy issues. Human Resources for Health [Internet]. 2003 [citado 10 noviembre 2010]; 1:4. Disponible en: http:// www.human-resources-health.com/content/1/1/4. 05.05.2009

20. PHANG ROMERO C. Reforma del sector salud y la política farmacéutica en Perú. Cad. Saúde Pública. 2002; 18(4): 1121-1138.

21. MEJÍA RESTREPO S, VÉLEZ ARANGO AL, BURITICÁ ARBOLEDA OC, ARANGO MEJÍA MC, DEL RÍO GÓMEZ JA. La política farmacéutica nacional en Colombia y la reforma de la seguridad social: acceso y uso racional de medicamentos. Cad. Saúde Pública. 2002; 18(4): 1025-1039.

22. DURÁN JE, MÉNDEZ CA. Implementación de la reforma de la salud: percepción del profesional químico farmacéutico. Cuad Med Soc (Chile) 2010; 50(2): 124-131.

Recepción: 25 de noviembre de 2010 Aprobación: 6 de mayo de 2011

Usted puede comentar éste y otros artículos publicados en la Revista Chilena de Salud Pública, enviando un correo electrónico a revistasp@med.uchile.cl 